\title{
Semantic Mash-Up Personal and Pervasive Learning Environments (SMupple)
}

\author{
Ahmet Soylu ${ }^{1}$, Fridolin Wild ${ }^{2}$, Felix Mödritscher ${ }^{3}$, and Patrick De Causmaecker ${ }^{1}$ \\ ${ }^{1}$ K. U. Leuven, Department of Computer Science, CODeS, iTec, Kortrijk, Belgium \\ \{Ahmet. Soylu, Patrick. DeCausmaecker\} @kuleuven-kortrijk.be \\ ${ }^{2}$ The Open University, Knowledge Media Institute, Milton Keynes, United Kingdom \\ f.wild@open.ac.uk \\ ${ }^{3}$ Vienna University of Economics and Business, Department of Information Systems, \\ Vienna, Austria \\ felix.moedritscher@wu.ac.at
}

\begin{abstract}
Personal Learning Environments have emerged as a complementary, even challenging, paradigm to Adaptive Learning Systems. We consider the mash-up era as an appropriate approach for a successful realization of digital personal learning environments. However, mash-ups are also accompanied by critical technical and usability challenges. In this paper, we try to identify some of these challenges and present our solution approach which results in Semantic Mash-up Personal and Pervasive Learning Environments (SMupple).
\end{abstract}

Keywords: Personal Learning Environments, Mash-up, Ontologies, Embedded Semantics, Workflows, Pervasive Computing.

\section{Introduction}

Adaptive Learning Systems (ALSs), in general, focuses on automatically, often intrusively, changing the system behavior, according to the learner's needs and other characteristics and aiming at adapting the learning material and its presentation. However, it is already apparent that it is not possible to predefine adaptation rules for all different usage contexts. Furthermore, Wild and his colleagues [1] claim that adaptation technologies take away experiences from end-users (learners) thus prohibiting the development of important competences. In this respect, Personal Learning Environments (PLEs) emerge as a complementary, even challenging, paradigm to the ALSs. Wild et al [1] value learning environment as an important aspect of the learning process and consider it as an output of learning rather than a mere input. Digital learning environments can be composed of different applications, artifacts, and people etc. The individual at the centre modifies this environment through interacting with it, intending to positively influence her social, self, methodological, and professional competences and to change her potentials for future action. In other words, a learner actively or passively creates her own personal learning environment. In short, one can argue that PLEs aim at replacing the physical learning environment while ALSs focus on replacing the instructor. 
Considering PLEs, learners acknowledge the abundance and variety of web applications, services and data sources to be used within their environments. Moreover, different technological devices, like mobile phones, digital media solutions, tablet PCs, intelligent household appliances, etc. are expected to be connected to the Web and serve their functionalities through embedded web servers or gateways coupled with the internal functions of available devices, possibly, with RESTful APIs [2]. This leads us to extend PLE paradigm to Personal and Pervasive Learning. Here, mash-up approaches enable users to design their ubiquitous and personal learning environments through combining functionalities and data available on the Web. However this leads to some challenges. In this paper, we identify these important challenges and present our solution approach, which builds on semantic technologies and, referring to [1], is called Semantic Mash-up Personal and Pervasive Learning Environments (SMupple).

\section{Approach}

We consider the mash-up paradigm to be crucial for realizing the PLE vision within the infinite space of the Web. However, before moving forward with our approach, we believe that a conceptual description of a personal learning environment and identification of basic requirements for a digital PLE shall be useful for situating important challenges. On a conceptual level, a user (learning) environment can be seen as space of entities, including people, artifacts, tools, learning objects etc., available to the learner. Each of these entities is attached with several possible activities; additionally composite activities and composite entities encompass several other entityactivity pairs and entities respectively. In that space learners derive their personal (sub-) environments, orchestrate member entities for their goals through maintaining data and interaction flows between these entities, and continuously refine the PLEs as a result of their activities and often through their own implicit formative assessment methods. A PLE can be further partitioned into disjoint or overlapping clusters with respect to varying goals of learners. Learners often shift their focus from one cluster to another according to their current goals. From this perspective, a mash-up personal and pervasive learning environment enables learners to construct their digital learning environments spanning various digital web resources and web-enabled devices encapsulated through widget like constructs.

Considering mash-ups, they can be created at client-side (i.e., at browser) or at web server-side. We identify two different types of mash-ups: (1) dashboard type (e.g., [1]), (2) box type (e.g., [3]). The former is usually created at the client-side where different applications are placed to the learner browser as widgets (all visible). Data and events can be moved from one widget to another one mainly through inter widget communication on the client, occasionally also through server-sided synchronization mechanisms. The latter mash-up type is usually created and provided by a server, combining the different applications into one single user experience (only the resulting application is visible). Data and events can be moved from one application to other application through server-sided synchronization mechanisms. The end product can also be used for developing other mash-ups of both types. 
With respect to above descriptions and by considering the existing implementations [4], we identified several challenges. These challenges and our approach is described in three tiers which is partially depicted in Fig. 1.

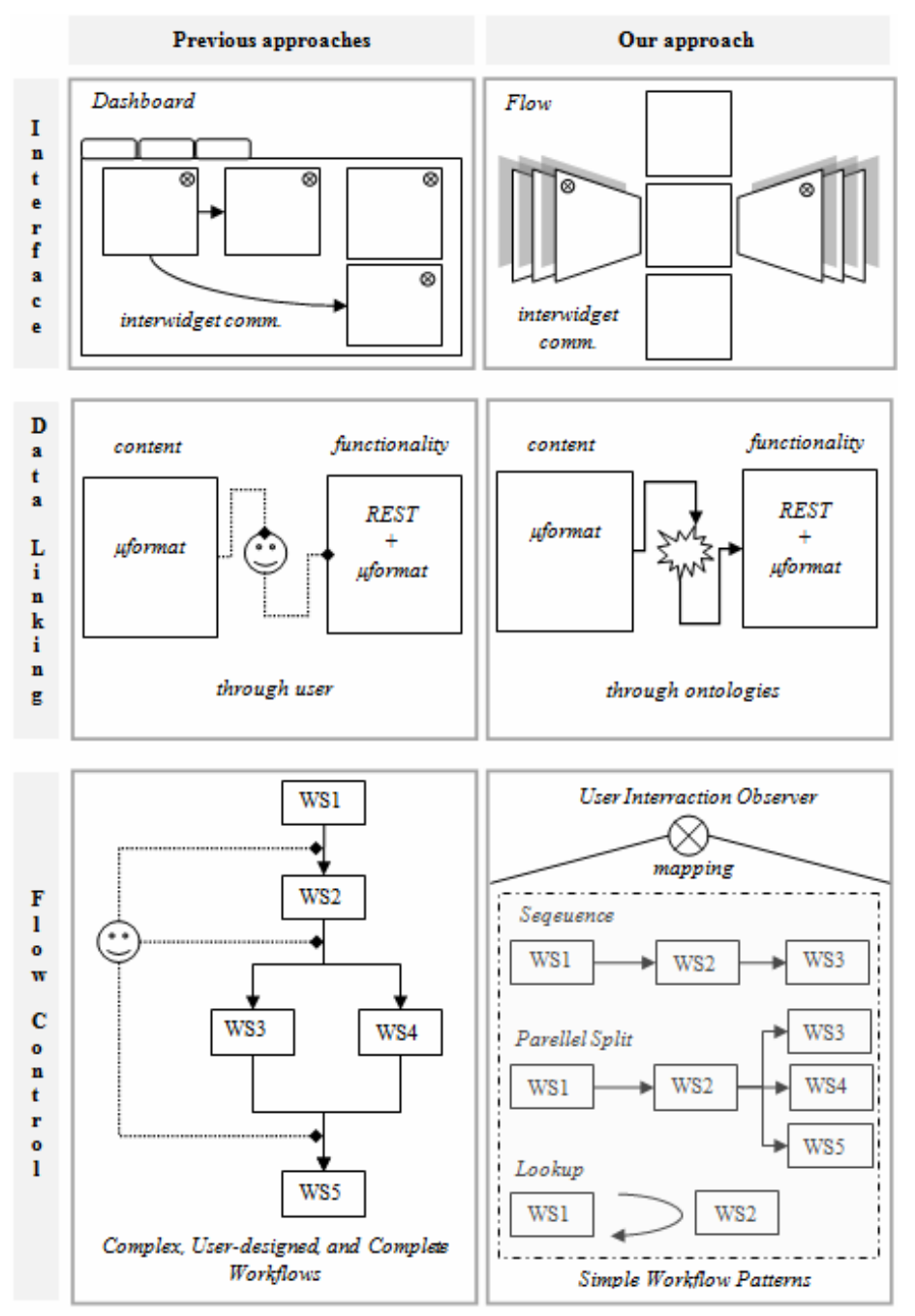

Fig. 1. Presentation and comparison of different approaches along the three tiers

Seven challenges have been identified each mapping to at least one tier: (1) composition/integration (services, applications and data), (2) inter widget communication. The first two challenges deal with data links between different applications, through server-sided synchronization or inter widget communication based on syntactic means, which is not sufficient for automated integration and composition of services leaving a huge burden to the end-user. Accordingly, injecting semantics through 
ontologies and embedded semantics technologies (e.g., microformats) may serve well for automated linking (e.g., [3, 5]). (3) Workflow management: this challenge is related to typical mash-up composition and requires users to define full workflows thus cognitively overloading the learner. Therefore, it is important to enable mash-up composition on the basis of incomplete workflows automatically generated through observing user interactions. (4) Environment awareness and control [6]: in physical environments users manage a limited number of entities with a relatively high awareness, however the Web offers an almost infinite amount of resources; therefore it is crucial to maintain awareness and control of one's space, so that the links between a learner and the environment stay tight. (5) Ease of orchestration: since the learner is confronted with more resources, learners should not experience a cognitive overload while managing the space. (6) Engaging learner experience: learners should feel comfortable through their experiences with PLEs. Hence identification and amalgamation of engaging and easy-to-use end-user design facilities and metaphors are required. (7) Adaptive guidance and support: this challenge is necessitated from the fact that learning process and end-user design of the environments requires adequate machine support, in terms of non-invasive adaptations, and recommendations. At this point, a formalized representation of the user's context through an ontology is promising with respect to "intelligent" guidance and end-user environment design. For the usability concerns, we approach a new type of mash-ups, a "flow" (see Fig. 1). Unlike dashboard type mash-ups, it tries to provide a reflection of the workflow among the widgets and the clustered nature of the learning environment.

We have elaborated on a scripting language and a design environment for realizing box like mash-ups addressing users ranging from experts to naïve. Our end-user tests, particularly on the interface mockup, have revealed that the mash-up paradigm is quite new, and hard to grasp for non-experts. Developing natural and easy-to-use design environments stands as a main challenge. However, apart from appropriates of design facilities, setting a smooth balance between machine and user control is required, so that users are not overloaded or not totally dominated by the machine. In that sense, we believe that automated data linking and workflow creation, as well as adaptive recommendations are more promising than strong, rule-based adaptation.

\section{References}

1. Wild, F., Mödritscher, F., Sigurdarson, S.E.: Designing for Change: Mash-Up Personal Learning Environments. eLearning Papers (9) (2008) ISSN: 1887-1542

2. Dillon, T., Talevski, A., Potdar, V., Chang, E.: Web of Things as a Framework for Ubiquitous Intelligence and Computing. In: Zhang, D., Portmann, M., Tan, A.-H., Indulska, J. (eds.) UIC 2009. LNCS, vol. 5585, pp. 1-10. Springer, Heidelberg (2009)

3. Sheth, A.P., Gomadam, K., Lathem, J.: SA-REST: Semantically Interoperable and Easierto-Use Services and Mashups. IEEE Internet Computing 11, 91-94 (2007)

4. Taivalsaari, A.: Mashware: The future of web applications. Sun Microsystems (2009)

5. Kopecky, J., Gomadam, K., Vitvar, T.: hRESTS: An HTML Microformat for Describing RESTful Web Services. In: International Conference on Web Intelligence and Intelligent Agent Technology (WI-IAT 2008), pp. 619-625 (2008)

6. Spiekermann, S.: User Control in Ubiquitous Computing: Design Alternatives and User Acceptance. Shaker Verlag, Aachen (2008) 